\title{
The dynamic evolutionary history of genome size in North American woodland salamanders
}

\begin{tabular}{|r|l|}
\hline Journal: & Genome \\
\hline Manuscript ID & gen-2016-0166.R1 \\
\hline Manuscript Type: & Article \\
\hline Date Submitted by the Author: & 31 -Oct-2016 \\
\hline Complete List of Authors: & $\begin{array}{l}\text { Newman, Catherine; Louisiana State University, Museum of Natural } \\
\text { Science; Louisiana State University, Department of Biological Sciences } \\
\text { Gregory, Ryan; University of Guelph, } \\
\text { Austin, Christopher; Louisiana State University, Museum of Natural } \\
\text { Science; Louisiana State University, Department of Biological Sciences }\end{array}$ \\
\hline Keyword: & C-value, Feulgen densitometry, <i>Plethodon serratus</i>, Plethodontidae \\
\hline &
\end{tabular}


The dynamic evolutionary history of genome size in North American woodland salamanders

$$
\text { Catherine E. Newman }{ }^{1,2, *} \text {, T. Ryan Gregory }{ }^{3} \text {, Christopher C. Austin }{ }^{1,2}
$$

${ }^{1}$ Museum of Natural Science, Louisiana State University, 119 Foster Hall, Baton Rouge,

Louisiana 70803, USA

${ }^{2}$ Department of Biological Sciences, Louisiana State University, Baton Rouge, Louisiana, USA

${ }^{3}$ Department of Integrative Biology, University of Guelph, Guelph, Ontario, Canada

* Corresponding author. Email: newma014@gmail.com, Phone: +1-225-578-5393 


\begin{abstract}
The genus Plethodon is the most species rich salamander genus in North America, and nearly half of its species face an uncertain future. It is also one of the most diverse families in terms of genome sizes, which range from $1 \mathrm{C}=18.2 \mathrm{pg}$ to $69.3 \mathrm{pg}$, or 5-20 times larger than the human genome. Large genome size in salamanders results in part from accumulation of transposable elements and is associated with various developmental and physiological traits. However, genome sizes have been reported for only 25\% of Plethodon species (14 of 55). We collected genome size data for $P$. serratus to supplement an ongoing phylogeographic study, reconstructed the evolutionary history of genome size in Plethodontidae, and inferred probable genome sizes for the 41 species missing empirical data. Results revealed multiple genome size changes in Plethodon; genomes of western Plethodon increased, whereas genomes of eastern Plethodon decreased, followed by additional decreases or subsequent increases. The estimated genome size of $P$. serratus was $21 \mathrm{pg}$. New understanding of variation in genome size evolution, along with genome size inferences for previously unstudied taxa, provide a foundation for future studies on the biology of plethodontid salamanders.
\end{abstract}

Keywords: C-value, Feulgen densitometry, Plethodon serratus, Plethodontidae 


\section{Introduction}

In most non-amphibian tetrapod families, genome sizes (haploid nuclear DNA content) tend to be fairly stable, varying up to $5 \mathrm{pg}[1 \mathrm{pg}=0.978$ gigabases $(\mathrm{Gb})]$ among species (Gregory 2016). However, amphibians show much larger variation in genome size within families: up to a $12 \mathrm{pg}$ difference within frog families and up to $96 \mathrm{pg}$ within families within salamanders (Gregory 2016). The exceptionally wide variation within salamander families suggests that major genome size changes have occurred independently among lineages (Jockusch 1997).

Plethodontidae is the most species rich salamander family, with 451 species in 28 genera (AmphibiaWeb 2016). Among salamander families, Plethodontidae also has the widest range of genome sizes (Herrick and Sclavi 2014). Genome size in salamanders is related to chromosome size, not chromosome number (Sessions 2008). In Plethodontidae, Batrachoseps and the neotropical genera have $2 n=26$ chromosomes; all other genera have $2 n=28$ chromosomes (Leon and Kezer 1978). The genomes of plethodontid salamanders contain much larger quantities of transposable elements (TEs) than are found in most other vertebrate clades, but within Plethodontidae, TE content does not appear to be correlated with genome size (Sun et al. 2012). This suggests that factors in addition to TE proliferation more strongly drive genome size evolution within the family Plethodontidae.

Genome size data play a critical role in designing effective laboratory methods of modern DNA sequence data collection methods for phylogenetic and phylogeographic studies. Large genome sizes limit the efficiency of genomic preparation techniques for next-generation sequencing data sets (Hodges et al. 2009; McCartney-Melstad et al. 2016), as well as subsequent assembly of highly repetitive sequences. Established laboratory protocols have been successfully 
used with salamanders with genome sizes below average for the order (though still large relative to non-salamander tetrapods) (Newman and Austin, in press). However, even those species yielded sequence data with lower read depth and percentage of on-target reads than comparable studies with other vertebrates, suggesting that species with even larger genomes may present additional challenges. Study design therefore depends on the genome size(s) of the species of interest. Unfortunately, of the 686 salamander species (AmphibiaWeb 2016), only 179 species (26\%) have published empirical genome size data (Gregory 2016).

Because of the wide variation in genome size, plethodontid salamanders in particular would benefit from a thorough phylogenetic and genome range assessment. The genus Plethodon is the most species rich and ecologically diverse salamander genus in North America, with 55 species. The backbone phylogeny of the major lineages of Plethodon is well-established (Highton and Larson 1979; Highton 1995), but some relationships among closely related species remain unresolved (Fisher-Reid and Wiens 2011; Highton et al. 2012). In addition, extreme morphological stasis within Plethodon likely masks identification of cryptic species (Highton 1995, Mueller et al. 2004; Wake 2009, Highton et al. 2012; Pelletier et al. 2015). Plethodon is thus an interesting and species rich lineage for phylogeographic and species delimitation research. Nonetheless, mitochondrial genes have been shown to be sometimes misleading in Plethodon (Fisher-Reid and Wiens 2011), and low numbers of nuclear loci are sometimes not sufficient to fully resolve relationships (e.g., Newman \& Austin 2015). Therefore, multilocus genetic data sets with hundreds to thousands of loci are often required, necessitating knowledge of genome size to choose appropriate techniques. However, only 14 of 55 Plethodon species have estimated genome sizes (Gregory 2016). 
Here, we estimate the genome size for $P$. serratus as part of an ongoing phylogeographic study of the species (Newman and Austin 2015). We incorporate our data with available genome size and genetic sequence data to reconstruct the evolutionary history of genome size in plethodontid salamanders under a maximum-likelihood (ML) framework and infer likely genome sizes for extant species that currently lack empirical estimates.

\section{Materials and Methods}

Genome size

Haploid genome size (1C-value) data for 14 Plethodon species and outgroup species $E$. bislineata, A. lugubris, E. eschscholtzii, D. quadramaculatus, and D. fuscus were downloaded from the Animal Genome Size Database (Gregory 2016) (Table S1). For species with multiple genome size estimates in the database, we used the median in downstream analyses rather than the mean to minimize bias due to potentially erroneously high genome size values reported in one study. In particular, the genome sizes for E. bislineata, D. quadramaculatus, $P$. glutinosus, and $P$. jordani reported in Bachmann (1970) are substantially larger than values reported for the same species in more recent studies (Olmo 1973, 1974; Mizuno and Macgregor 1974; Hally et al. 1986; Sessions and Larson 1987; Licht and Lowcock 1991). This is potentially due to the use of a higher than usual genome size for Rana pipiens as a reference to convert relative values to absolute (Olmo 1974). However, because estimates of genome size do vary within species, we chose to incorporate but minimize the bias, rather than exclude the unusually high data points. Results from the same analyses using mean instead of median genome sizes were not qualitatively different (data not shown). 
In addition, we generated the first estimate of genome size for $P$. serratus as part of a phylogeographic and phylogenomic study of the species. Blood smears were made for five individuals of $P$. serratus collected at Sicily Island Hills Wildlife Management Area in Louisiana. Blood was collected on site from the caudal vein of live salamanders after removing the tail tip for a future genetic resource. All blood and tissue samples were deposited in the Louisiana State University Museum of Natural Science Herpetology Collection. Field work was conducted in accordance with the Guide for the Care and Use of Laboratory Animals (National Research Council 2011), under a protocol approved by the Institutional Animal Care and Use Committee (IACUC) of Louisiana State University (permit number 13-060).

Genome size was estimated for P. serratus using the Feulgen Image Analysis Densitometry (FIAD) method described in detail by Hardie et al. (2002). Air-dried blood smears were post-fixed overnight in 85:10:5 methanol : formalin : glacial acetic acid, rinsed in running tap water, and then hydrolyzed in $5 \mathrm{~N} \mathrm{HCl}$ for 2 hours, followed by staining in freshly-prepared Schiff reagent for 2 hours. The slides were passed through a series of metabisulfite and distilled water rinses before being dried and stored in the dark until analysis. Genome size was estimated by using the Bioquant Life Science image analysis package along with a Leica DM2500 microscope using a 63x oil-immersion lens connected to a Retiga EXi digital camera. Integrated optical densities (IODs) were measured for at least 50 nuclei per specimen and converted to absolute genome size by comparison with nuclei of the salamander Ambystoma jeffersonianum $(1 \mathrm{C}=28.8 \mathrm{pg} ;$ Licht and Lowcock 1991). Chicken and rainbow trout blood were also included as internal checks of the Feulgen staining.

Phylogenetic analysis 
Genetic sequence data from several previously published studies (Wiens et al. 2006; Vieites et al. 2007; Bonett et al. 2009; Fisher-Reid and Wiens 2011; Martin et al. 2015) were downloaded from GenBank (Benson et al. 2013) http://www.ncbi.nlm.nih.gov/genbank, retrieved 19 Feb. 2016 for 55 species in the family Plethodontidae, including 50 Plethodon species (Table S2). The concatenated alignment contained a total of 4,057 bp and included the following nuclear loci: BDNF (707 bp), GAPD (644 bp), ILF3 (281 bp), Mlc2a (253 bp), POMC (481 bp), RAG-1 (1,467 bp) and RHO (224 bp). For each locus, sequences were aligned in Geneious v.6.0.5 using the ClustalW algorithm. We generated two data sets for downstream analyses: one including all species, and another including only species with empirical genome size data.

Intentionally, our genetic data set overlapped almost entirely with the nuclear-only data set in Fisher-Reid \& Wiens (2011), the most recent multilocus plethodontid phylogenetic study with extensive taxon sampling within Plethodon. We thus implemented the same partitioning scheme, models of nucleotide evolution, and software settings described in the previous paper to generate a phylogeny under a Bayesian framework in MrBayes v.3.2.6 (Ronquist et al. 2012). We conducted two MCMC runs of 6 million generations, sampling every 1000 generations. Convergence was assessed in Tracer v.1.6 (Rambaut and Drummond 2007) by ensuring effective sample sizes $(\mathrm{ESSs})>200$. The first $10 \%$ of samples were discarded as burn-in.

\section{Ancestral character reconstruction}

Genome sizes of ancestral nodes (most recent common ancestor, MRCA) were estimated in a likelihood framework under a Brownian motion model using the phytools package v.0.5.20 (Revell 2012) in R v.3.2.1 (R Core Team 2015). This method of ancestral character 
reconstruction (ACR) allows for missing character data for some tips on the phylogeny and, in addition to reconstructing ancestral characters, also estimates values for the tips missing empirical data. To assess whether or not the large amount of missing character data $(73 \%)$ influenced reconstruction of ancestral states, we performed the same analysis using a phylogeny comprised only of species with empirical genome size data. Lastly, we ran the analysis on the full phylogeny but excluding the empirical genome size data for $P$. serratus to test the accuracy of the method's character state estimation for extant taxa.

\section{Results}

The estimated haploid genome size for P. serratus was $21.01 \mathrm{pg} \pm 0.77$ SE (range: 19.23 - $24.21 \mathrm{pg}$ ) (Table 1, Fig. 1), within the range of previously available data for the $P$. cinereus clade (18.20 - 26.20 pg). Partitioned Bayesian analysis of both sets of taxa yielded strong support (posterior probability $[P] \geq 0.9$ ) for all major clades: Plethodontinae, genus Plethodon, western Plethodon, eastern Plethodon, and, within eastern Plethodon, the P. cinereus, P. wehrlei + P. welleri, and P. glutinosus groups (Fig. S1). The sister relationship of the P. cinereus group to the remainder of eastern Plethodon is also strongly supported. This topology is congruent with the previous study that used the same genetic data and analyses (Fisher-Reid and Wiens 2011).

The genome size of the MRCA of all Plethodon was estimated to be $33.82 \mathrm{pg}$ (Table 2, Fig. 2), which falls slightly above the median empirical genome size of all salamanders (30.07 pg). Within Plethodon, the estimated genome size was smaller for the MRCA of the eastern Plethodon (25.71 pg) but larger for the MRCA of the western Plethodon (38.71 pg). It is thus likely that, relative to salamanders as a group, modest genome size is the ancestral state for Plethodon, with a subsequent increase in genome size among the western species and decrease 
among the eastern species. Within the eastern Plethodon, estimated MRCA genome sizes ranged from $22.22 \mathrm{pg}$ for the $P$. cinereus group to 29.07 for the $P$. glutinosus group. This suggests that the $P$. glutinosus group has undergone expansion of genome size since diverging from the remainder of the eastern Plethodon. Genome size appears to have been relatively stable along the lineage leading to Plethodon before diversification, as the genome size of the MRCA of all Plethodontinae was estimated to be 32.63 .

There were no qualitative differences between estimated genome sizes of ancestral nodes when the ACR analysis was run on the phylogeny including only species with empirical genome size data and analysis with the full phylogeny (Table S3; Figs. S3, S4). When the ACR analysis was run without the empirical $P$. serratus genome size data, the genome size of $P$. serratus was estimated to be $21.58 \mathrm{pg}$, consistent with the empirical median value of $21.01 \mathrm{pg}$. Genome sizes for extant species estimated from empirical data sets including and omitting $P$. serratus were highly similar (Table 3).

\section{Discussion}

Salamanders of the genus Plethodon appear to have undergone multiple contractions and expansions of genome size. Consistent with previous studies (Sessions and Larson 1987; Kraaijeveld 2010), our results suggest a genome size contraction preceding or concurrent with diversification of the eastern species. Some researchers (Kraaijeveld 2010; Herrick and Sclavi 2014) have used this result as an example of an evolutionary radiation following a reduction in genome size. However, Kozak et al. (2006) found that only the P. glutinosus group underwent a rapid radiation that produced a significantly higher number of extant lineages than expected. Our ACR analysis reconstructed a genome size expansion along the lineage leading to the $P$. 
glutinosus group, suggesting that an expansion, rather than a reduction, in genome size is associated with the only radiation within Plethodon. On a broader phylogenetic scale, the family Plethodontidae has some of the smallest genome sizes of all salamanders and also the highest number of species, suggesting a negative correlation between genome size and speciation rate (Herrick and Sclavi 2014). However, our data of the genus Plethodon suggest a more complex relationship between genome size and speciation at this finer scale.

The three major lineages within the eastern Plethodon appear to be undergoing independent evolution of genome size, with one lineage showing a further reduction $(P$. cinereus group), one lineage showing an expansion ( $P$. glutinosus group), and one lineage showing no change ( $P$. wehrlei $+P$. welleri group). Within each group, there is little variation in genome size. The causes of genome size variation in salamanders are not yet well understood. However, various hypotheses have been proposed and explored, including life history constraints (Gregory 2002) and developmental constraints (Sessions and Larson 1987; Jockusch 1997). In addition, it has been proposed that smaller genome size is associated with younger lineages in salamanders, both among families and among genera within a family (Herrick and Sclavi 2014). Our results did not show this pattern for clades within the genus Plethodon. Species in the P. cinereus group have smaller genome sizes than species in the $P$. glutinosus group, yet the $P$. glutinosus group is younger (Martin et al. 2015). Further exploration of the potential factors underlying genome size variation in Plethodon is beyond the scope of this study.

We also highlight the need for a broad examination of genome size in Plethodon through collection of additional empirical genome size data. Of the 55 recognized species of Plethodon, empirical data are now currently available for only 15 species, or $28 \%$. Furthermore, of the available data, only two data points were obtained in the past 15 years: one $P$. cinereus estimate 
(Mueller et al. 2008) and P. serratus (this study). All other Plethodon genome size data were collected between 1968-1998, using several different methods. While any substantial deviation from the estimated values in our study would be unexpected, it is difficult to assess the biological significance of the variation in genome sizes among the eastern Plethodon groups without more complete information about intra-group variation among species. In addition, our empirical data for $P$. serratus show that even within a single population of a species, genome size estimates can vary $(19.23-24.21 \mathrm{pg})$. A promising approach to collecting not only genome size data but also information about genome content is shotgun sequencing alongside chromosome capture and sequencing, as was recently used to estimate the genome size of Ambystoma mexicanum and explore the nature of repetitive elements of the genome (Keinath et al. 2015).

Full evaluation of the evolution of genome size in Plethodon requires a larger data set consisting of appropriate multilocus sequence data and genome size data for multiple individuals of each Plethodon species - with both genetic and genome size data for each individual. Our results are consistent with previous studies and also consistent with our expectations based on phylogeny - i.e., Plethodon species show clear genome size differentiation by clade - but the omission of within-species variation may mask other patterns or miss important outliers. For example, Gregory (2002) notes that while most Desmognathus undergo metamorphosis from aquatic larvae and possess the smallest genomes of salamanders, the one Desmognathus species now known to be direct developing (D. aeneus) has an unknown genome size. Hypothetically, if genome size is indeed associated with developmental cycle, the ACR method would fail to recognize D. aeneus as an outlier within its clade of small-genome Desmognathus. Further, inferences drawn from ACR analysis are based on the assumption that a Brownian motion model is a reasonable fit for genome size evolution. A Brownian motion model might not be 
appropriate if, for example, genome size is under strong selection pressure (Elliot and Mooers 2014).

Our results reveal variation in the direction of genome size evolution among lineages within Plethodon. In particular, the genome size expansion at the base of the $P$. glutinosus group suggests that caution is needed when ascribing a particular pattern of evolution to higher-level clades. We also highlight the interdependence between studies of genome size evolution and the fields of phylogeography and population genetics. The new genome size estimates presented here will facilitate much-needed phylogeographic and population genetic studies of this system, and new information on patterns of genome size evolution in Plethodon will contribute to exploration of factors underlying large genomes.

The most species rich plethodontid genus, Plethodon, is also highly threatened. Of the 44 species that have been assessed and ranked by the IUCN Red List (excluding "Data Deficient" species), 20 are ranked at least Near-Threatened (IUCN 2016). In addition, many species have very small geographic distributions, restricted to one or a few mountain peaks. Future research on the evolution of genome size in plethodontids will enhance our understanding of the associations between genome size and other aspects of a salamander's biology - including potential adaptive significance of genome size.

\section{Acknowledgements}

We thank Zachary Rodriguez, Geneveive Mount, Seth Parker, AJ Turner, and Subir Shakya for field work help. We also thank Rachel Mueller and Rebecca Chong for invaluable advice on sample collection and preparation. All tissue collection was conducted under the following state collecting permits for Louisiana: Scientific Collecting Permit LNHP-16-003 and 
Wildlife Division Special Use Permit to Conduct Research on Wildlife Management Areas \#WL-Research-2016-05. Collecting by the authors was conducted in accordance with IACUC protocol 13-060 approved at Louisiana State University. This work was funded by a Natural Sciences and Engineering Research Council of Canada (NSERC) Discovery Grant to TRG and by National Science Foundation grants DEB 1405665 to CEN and CCA and DEB 1146033 to CCA. 


\section{References}

AmphibiaWeb. 2016. AmphibiaWeb: information on amphibian biology and conservation. Berkeley, CA. Available from http://amphibiaweb.org [accessed 18 July 2016].

Bachmann, K. 1970. Feulgen slope determinations of urodele nuclear DNA amounts. Histochemie 22(4): 289-293. doi:10.1007/BF00277456.

Benson, D.A., Cavanaugh, M., Clark, K., Karsch-Mizrachi, I., Lipman, D.J., Ostell, J., and Sayers, E.W. 2013. GenBank. Nucleic Acids Res. 41(212): D36-42. doi:10.1093/nar/gks1195.

Bonett, R.M., Chippindale, P.T., Moler, P.E., Van Devender, R.W., and Wake, D.B. 2009. Evolution of gigantism in amphiumid salamanders. PLoS One 4(5): e5615. doi:10.1371/journal.pone.0005615.

Elliot, M.G., and Mooers, A.O. 2014. Inferring ancestral states without assuming neutrality or gradualism using a stable model of continuous character evolution. BMC Evol. Biol. 14: 226.

Fisher-Reid, M.C., and Wiens, J.J. 2011. What are the consequences of combining nuclear and mitochondrial data for phylogenetic analysis? Lessons from Plethodon salamanders and 13 other vertebrate clades. BMC Evol. Biol. 11: 300. doi:10.1186/1471-2148-11-300.

Gregory, T.R. 2002. Genome size and developmental complexity. Genetica 115(1): 131-146. doi:10.1023/A:1016032400147.

Gregory, T.R. 2016. Animal Genome Size Database. Available from http://genomesize.com [accessed 23 May 2016]. 
Hally, M.K., Rasch, E.M., Mainwaring, H.R., and Bruce, R.C. 1986. Cytophotometric evidence of variation in genome size of desmognathine salamanders. Histochemistry 85(3): 185-192. doi:10.1007/BF00494802.

Hardie, D.C., Gregory, T.R., and Hebert, P.D.N. 2002. From pixels to picograms: a beginners' guide to genome quantification by Feulgen Image Analysis Densitometry. J. Histochem. Cytochem. 50(6): 735-749. doi:10.1177/002215540205000601.

Herrick, J., and Sclavi, B. 2014. A new look at genome size, evolutionary duration and genetic variation in salamanders. Comptes Rendus - Palevol 13(7): 611-621. doi:10.1016/j.crpv.2014.06.002.

Highton, R. 1995. Speciation in eastern North American salamanders of the genus Plethodon. Annu. Rev. Ecol. Syst. 26(1995): 579-600.

Highton, R., Hastings, A.P., Palmer, C., Watts, R., Hass, C.A., Culver, M., and Arnold, S.J. 2012. Concurrent speciation in the eastern woodland salamanders (Genus Plethodon): DNA sequences of the complete albumin nuclear and partial mitochondrial $12 \mathrm{~s}$ genes. Mol. Phylogenet. Evol. 63(2): 278-290. doi:10.1016/j.ympev.2011.12.018.

Highton, R., and Larson, A. 1979. The genetic relationships of the salamanders of the genus Plethodon. Syst. Biol. 28(4): 579-599. doi:10.2307/sysbio/28.4.579.

Hodges, E., Rooks, M., Xuan, Z., Bhattacharjee, A., Benjamin Gordon, D., Brizuela, L., Richard McCombie, W., and Hannon, G.J. 2009. Hybrid selection of discrete genomic intervals on custom-designed microarrays for massively parallel sequencing. Nat. Protoc. 4(6): 960-974. doi:10.1038/nprot.2009.68. 
IUCN. 2016. IUCN Red List of Threatened Species. Available from http://iucnredlist.org [accessed 19 July 2016].

Jockusch, E.L. 1997. An evolutionary correlate of genome size change in plethodontid salamanders. Proc. R. Soc. B 264(1381): 597-604. doi:10.1098/rspb.1997.0085.

Kleinath, M.C., Timoshevskiy, V.A., Timoshevskaya, N.Y., Tsonis, P.A., Voss, S.R., and Smith, J.J. 2015. Initial characterization of the large genome of the salamander Ambystoma mexicanum using shotgun and laser capture chromosome sequencing. Sci. Rep. 5: 16413.

Kozak, K.H., Weisrock, D.W., and Larson, A. 2006. Rapid lineage accumulation in a nonadaptive radiation: phylogenetic analysis of diversification rates in eastern North American woodland salamanders (Plethodontidae: Plethodon). Proc. R. Soc. B 273(1586): 539-46. doi:10.1098/rspb.2005.3326.

Kraaijeveld, K. 2010. Genome size and species diversification. Evol. Biol. 37(4): 227-233. doi:10.1007/s11692-010-9093-4.

Leon, P.E., and Kezer, J. 1978. Localization of 5S RNA genes on chromosomes of plethodontid salamanders. Chromosoma 65: 213-230.

Licht, L.E., and Lowcock, L.A. 1991. Genome size and metabolic rate in salamanders. Comp. Biochem. Physiol. 100(1): 83-92. doi:10.1016/0305-0491(91)90089-V.

Martin, S.D., Shepard, D.B., Steffen, M.A., Phillips, J.G., and Bonett, R.M. 2015. Biogeography and colonization history of plethodontid salamanders from the Interior Highlands of eastern North America. J. Biogeogr.: 410-422. doi:10.1111/jbi.12625.

McCartney-Melstad, E., Mount, G.G., and Shaffer, H.B. 2016. Exon capture optimization in 
large-genome amphibians. Mol. Ecol. Resour. doi:10.1111/1755-0998.12538.

Mizuno, S., and Macgregor, H.C. 1974. Chromosomes, DNA sequences, and evolution in salamanders of the genus Plethodon. Chromosoma 48(3): 239-296. doi:10.1007/BF00326507.

Mueller, R.L., Gregory, T.R., Gregory, S.M., Hsieh, A., and Boore, J.L. 2008. Genome size, cell size, and the evolution of enucleated erythrocytes in attenuate salamanders. Zoology 111(3): 218-30. doi:10.1016/j.zool.2007.07.010.

Mueller, R.L., Macey, J.R., Jaekel, M., Wake, D.B., and Boore, J.L. 2004. Morphological homoplasy, life history evolution, and historical biogeography of plethodontid salamanders inferred from complete mitochondrial genomes. Proc. Natl. Acad. Sci. 101(38): 1382013825. doi:10.1073/pnas.0405785101.

National Research Council. 2011. Guide for the Care and Use of Laboratory Animals. National Academies Press, Washington, D.C. doi:10.17226/12910.

Newman, C.E., and Austin, C.C. 2015. Thriving in the cold: glacial expansion and post-glacial contraction of a temperate terrestrial salamander (Plethodon serratus). PLoS One 10: e0130131. doi:10.1371/journal.pone.0130131.

Newman, C.E., and Austin, C.C. In press. Sequence capture and next-generation sequencing of ultraconserved elements in a large-genome salamander. Mol. Ecol. doi:10.1111/mec.13909.

Olmo, E. 1973. Quantitative variations in the nuclear DNA and phylogenesis of the Amphibia. Caryologia 25(April 2015): 43-68. doi:10.1080/00087114.1973.10796525.

Olmo, E. 1974. Further data on the genome size in the urodeles. Boll. Di Zool. 41(1): 29-33. 
doi:10.1080/11250007409430082.

Pelletier, T.A., Crisafulli, C., Wagner, S., Zellmer, A.J., and Carstens, B.C. 2015. Historical species distribution models predict species limits in western Plethodon salamanders. Syst. Biol. 64(6): 909-925. doi:10.1093/sysbio/syu090.

R Core Team. 2015. R: A Language and Environment for Statistical Computing. Vienna, Austria.

Rambaut, A., and Drummond, A.J. 2007. Tracer v.1.4.

Revell, L.J. 2012. phytools: an R package for phylogenetic comparative biology (and other things). Methods Ecol. Evol. 3(2): 217-223. doi:10.1111/j.2041-210X.2011.00169.x.

Ronquist, F., Teslenko, M., van der Mark, P., Ayres, D.L., Darling, A., Höhna, S., Larget, B., Liu, L., Suchard, M.A., and Huelsenbeck, J.P. 2012. MrBayes 3.2: efficient Bayesian phylogenetic inference and model choice across a large model space. Syst. Biol. 61(3): 53942. doi:10.1093/sysbio/sys029.

Sessions, S.K. 2008. Evolutionary cytogenetics in salamanders. Chromosom. Res. 16: 183-201. doi:10.1007/s10577-007-1205-3.

Sessions, S.K., and Larson, A. 1987. Developmental correlates of genome size in plethodontid salamanders and their implications for genome evolution. Evolution. 41(6): 1239-1251.

Sun, C., Shepard, D.B., Chong, R.A., López Arriaza, J., Hall, K., Castoe, T.A., Feschotte, C., Pollock, D.D., and Mueller, R.L. 2012. LTR retrotransposons contribute to genomic gigantism in plethodontid salamanders. Genome Biol. Evol. 4(2): 168-83. doi:10.1093/gbe/evr139. 
Vieites, D.R., Min, M.-S., and Wake, D.B. 2007. Rapid diversification and dispersal during periods of global warming by plethodontid salamanders. Proc. Natl. Acad. Sci. 104(50): 19903-7. doi:10.1073/pnas.0705056104.

Wake, D.B. 2009. What salamanders have taught us about evolution. Annu. Rev. Ecol. Evol. Syst. 40: 333-352. doi:http://dx.doi.org/10.1146/annurev.ecolsys.39.110707.173552.

Wiens, J.J., Engstrom, T.N., and Chippindale, P.T. 2006. Rapid diversification, incomplete isolation, and the "speciation clock" in North American salamanders (Genus Plethodon): testing the hybrid swarm hypothesis of rapid radiation. Evolution. 60(12): 2585-603. 
Table 1. Genome size estimates for P. serratus as determined by Feulgen image analysis densitometry.

\begin{tabular}{|l|c|c|c|c|}
\hline \multicolumn{1}{|c|}{ Sample ID } & IOD & Genome size (pg) & Magnification \\
\hline \multicolumn{4}{|c|}{ Plethodon serratus } \\
\hline LSUMZ H-21468a & 5644.15 & 22.14 & $63 \mathrm{x}$ \\
\hline LSUMZ H-21469 & 6170.92 & 24.21 & $63 \mathrm{x}$ \\
\hline LSUMZ H-21470 & 5007.92 & 19.64 & $63 \mathrm{x}$ \\
\hline LSUMZ H-21471 & 5371.06 & 21.07 & $63 \mathrm{x}$ \\
\hline LSUMZ H-21472 & 5048.16 & 19.80 & $63 \mathrm{x}$ \\
\hline & 4902.28 & 19.23 & $63 \mathrm{x}$ \\
\hline Ambystoma jeffersonianum & 7342.36 & 28.80 & $63 \mathrm{x}$ \\
\hline Oncorhynchus mykiss & 2787.56 & 2.33 & $100 \mathrm{x}$ \\
\hline Gallus domesticus & 1495.93 & 1.25 & $100 \mathrm{x}$ \\
\hline
\end{tabular}

* Integrated optical density (IOD) 
Table 2. Genome size for ancestral nodes, estimated by ACR.

\begin{tabular}{|c|c|c|}
\hline Node & Clade Name & Genome size (pg) \\
\hline 1 & Plethodontinae & 32.63 \\
\hline 2 & - & 32.49 \\
\hline 3 & Desmognathus & 17.65 \\
\hline 4 & Plethodon & 33.82 \\
\hline 5 & Western Plethodon & 38.71 \\
\hline 6 & Eastern Plethodon & 25.71 \\
\hline 7 & P. cinereus group & 22.22 \\
\hline 8 & - & 25.99 \\
\hline 9 & P. wehrlei $+P$. welleri group & 24.96 \\
\hline 10 & $P$. glutinosus group $+P$. websteri & 26.77 \\
\hline 11 & P. glutinosus group & 29.07 \\
\hline 12 & - & 28.74 \\
\hline 13 & - & 29.84 \\
\hline
\end{tabular}

Note: Node numbers correspond to Fig. 2. 
Table 3. Empirical and estimated genome sizes for all species included in the full phylogeny.

\begin{tabular}{|c|c|}
\hline Species & Genome size (pg) \\
\hline \multicolumn{2}{|c|}{ Eastern Plethodon: P. cinereus group } \\
\hline P. cinereus & 22.64 \\
\hline P. electromorphus & 21.25 \\
\hline P. hoffmani & 21.40 \\
\hline P. hubrichti & 21.67 \\
\hline P. nettingi & 21.51 \\
\hline P. richmondi & 20.65 \\
\hline P. serratus & 21.01 \\
\hline P. shenandoah & 18.20 \\
\hline P. virginia & 21.42 \\
\hline \multicolumn{2}{|c|}{ Eastern Plethodon: $P$. wehrlei $+P$. welleri group } \\
\hline P. angusticlavius & 24.14 \\
\hline P. dorsalis & 23.50 \\
\hline P. punctatus & 24.37 \\
\hline$P$. ventralis & 23.50 \\
\hline P. wehrlei & 24.20 \\
\hline P. welleri & 22.60 \\
\hline \multicolumn{2}{|c|}{ Eastern Plethodon: P. glutinosus group } \\
\hline P. albagula & 29.40 \\
\hline P. amplus & 28.32 \\
\hline P. aureolus & 28.43 \\
\hline P. caddoensis & 30.82 \\
\hline P. chattahoochee & 28.32 \\
\hline P. cheoah & 28.32 \\
\hline P. chlorobryonis & 28.32 \\
\hline P. cylindraceus & 28.32 \\
\hline
\end{tabular}




\begin{tabular}{|l|l|}
\hline P. fourchensis & 32.09 \\
\hline P. glutinosus & $\mathbf{2 8 . 0 0}$ \\
\hline P. grobmani & 29.40 \\
\hline P. jordani & $\mathbf{2 7 . 8 0}$ \\
\hline P. kentucki & 28.92 \\
\hline P. kiamichi & 29.40 \\
\hline P. kisatchie & 29.40 \\
\hline P. longicrus & 28.62 \\
\hline P. meridianus & 28.02 \\
\hline P. metcalfi & 28.02 \\
\hline P. mississippi & 29.40 \\
\hline P. montanus & 28.02 \\
\hline P. ocmulgee & 29.40 \\
\hline P. oconaluftee & 28.32 \\
\hline P. ouachitae & $\mathbf{3 3 . 7 0}$ \\
\hline P. petraeus & 28.74 \\
\hline P. savannah & 29.40 \\
\hline P. sequoyah & 29.40 \\
\hline P. shermani & 28.32 \\
\hline P. teyahalee & 28.32 \\
\hline P. variolatus & 28.32 \\
\hline P. websteri & 26.77 \\
\hline P. yonahlossee & $\mathbf{3 0 . 7 5}$ \\
\hline & $\mathbf{3 3 . 6 3}$ \\
\hline P. elongatus & 67.04 \\
\hline P. idahoensis & $\mathbf{6 9 . 3 0}$ \\
\hline P. vandykei & $\mathbf{3 8 . 0 5}$ \\
\hline P. vehiculum & \\
\hline
\end{tabular}




\begin{tabular}{|l|l|}
\hline \multicolumn{2}{|c|}{ Other Plethodontidae } \\
\hline Eurycea bislineata & 26.92 \\
\hline Aneides lugubris & 42.77 \\
\hline Ensatina eschscholtzii & 38.87 \\
\hline Desmognathus quadramaculatus & 17.19 \\
\hline Desmognathus fuscus & 16.17 \\
\hline
\end{tabular}




\section{Figure Legends}

Fig. 1. Photomicrographs of Feulgen-stained erythrocyte nuclei from the salamanders A) Ambystoma jeffersonianum $(1 \mathrm{C}=28.8 \mathrm{pg})$, and $\mathrm{B})$ Plethodon serratus $(1 \mathrm{C}=21.0 \mathrm{pg})$. Images taken under $63 \mathrm{x}$ magnification, scale bar $=20 \mu \mathrm{m}$.

Fig. 2. ML reconstruction of extant and ancestral genome sizes in Plethodontidae. Phylogeny: Bayesian majority-rule consensus tree with all 55 species. For taxon names, red + asterisk: species with empirical genome size data, black: genome size estimated by ACR. Node labels correspond to Table 2. Inset photograph of LSUMZ 98343 (credit: CCA) from Kisatchie National Forest, Louisiana. 

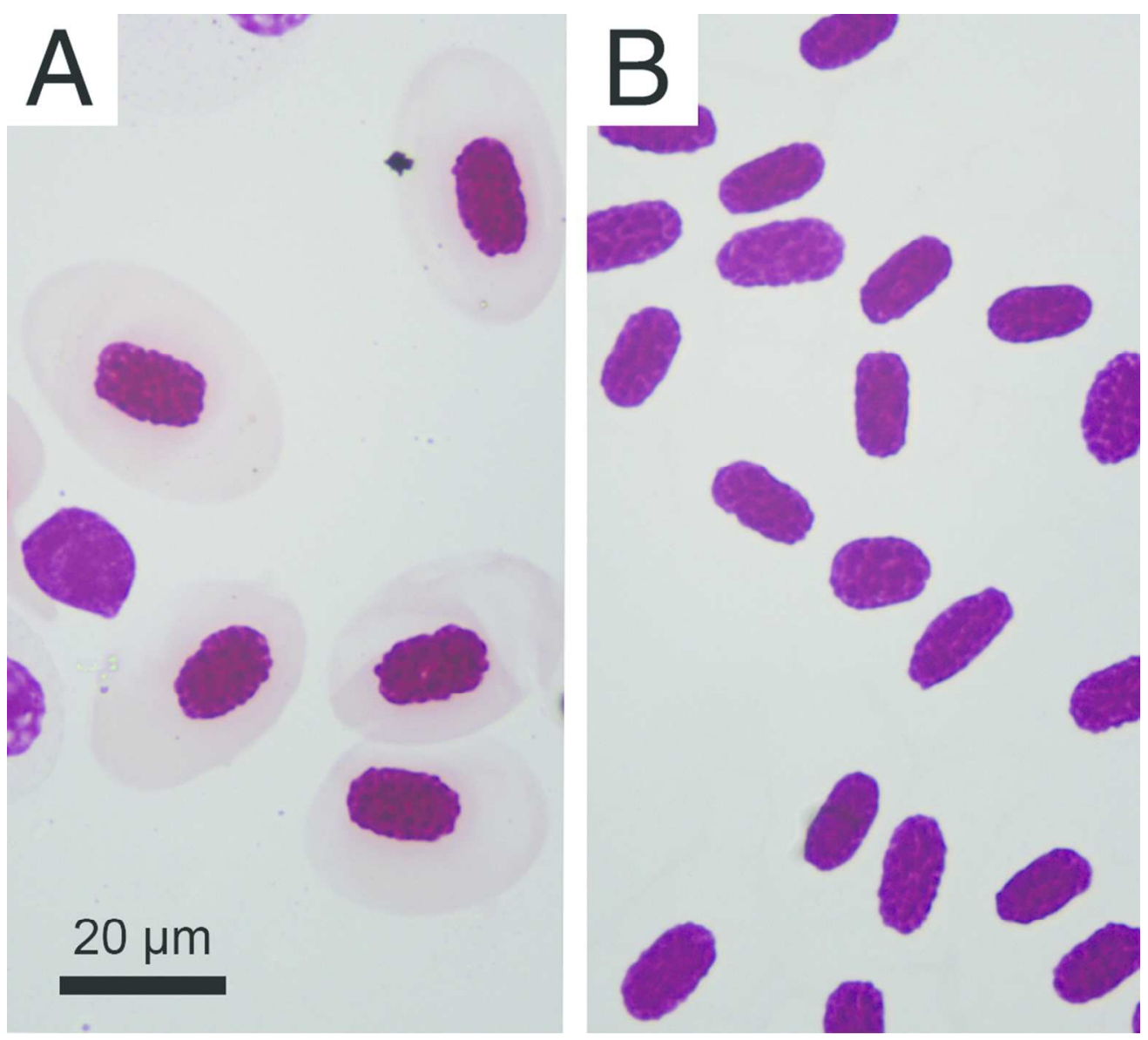

Photomicrographs of Feulgen-stained erythrocyte nuclei from the salamanders A) Ambystoma jeffersonianum $(1 \mathrm{C}=28.8 \mathrm{pg})$, and $\mathrm{B})$ Plethodon serratus $(1 \mathrm{C}=21.0 \mathrm{pg})$. Images taken under $63 \mathrm{x}$ magnification, scale bar $=20 \mu \mathrm{m}$.

Fig. 1

$95 \times 86 \mathrm{~mm}(300 \times 300$ DPI $)$ 


\section{Page 27 of 38}

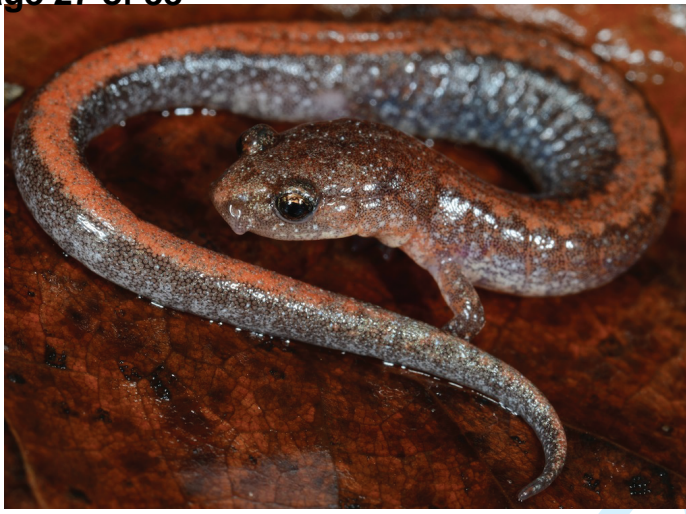

Plethodon serratus
Genome

11

13

$P$ P. kentucki
$P$ Plutinosus $P$ sequoyah P. mississippi $P$. savannah $P$. ocmulgee ㄱ. kisatchie P. kiamichi

$P$. grobmani $P$. albagula P. yonahlossee

$P$. petraeus

$P$. longicrus

12

$P$. aureolus

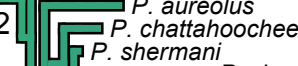

P. cheoah

P P. variolatus

P. amplus

P. cylindraceus

chlorobryonis

$P$. oconaluftee

5 . teyahalee P. montanus

P P. metcalfi

ك P. meridianus

$P$. jordani *

P. websteri

6

8

4

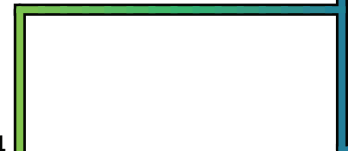

1

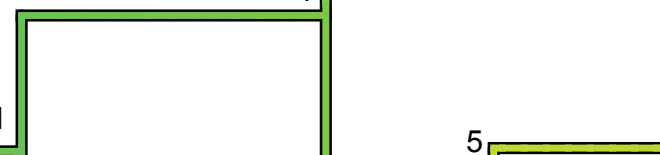

5

P. wehrlei *

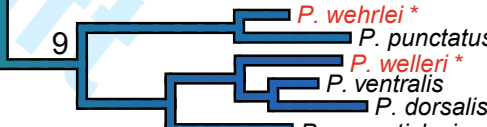
P. dorsalis

P. wehrlei + $P$. welleri group

$P$. cinereus group
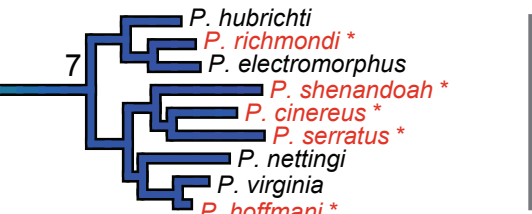

doah * *

P. glutinosus group

elongatus * P. vandykei *

\section{P. idahoensis}

D. quadramaculatus *

\section{3}

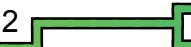

Antips.thme Eurycea bislineata * 


\section{Online Supplementary Material, Figure Legends}

Fig. S1. Bayesian majority-rule consensus tree with all 55 species. Nodes supported by Bayesian posterior probability $\geq 0.9$ are indicated by asterisk. Otherwise, posterior probability is noted.

Fig. S2. Bayesian majority-rule consensus tree with only species with empirical genome size data. Nodes supported by Bayesian posterior probability $\geq 0.9$ are indicated by asterisk. Otherwise, posterior probability is noted.

Fig. S3. Node numbers on Bayesian majority-rule consensus tree with all 55 species. Node numbers correspond to Table S3.

Fig. S4. Node numbers on Bayesian majority-rule consensus tree with only species with empirical genome size data. Node numbers correspond to Table S3. 


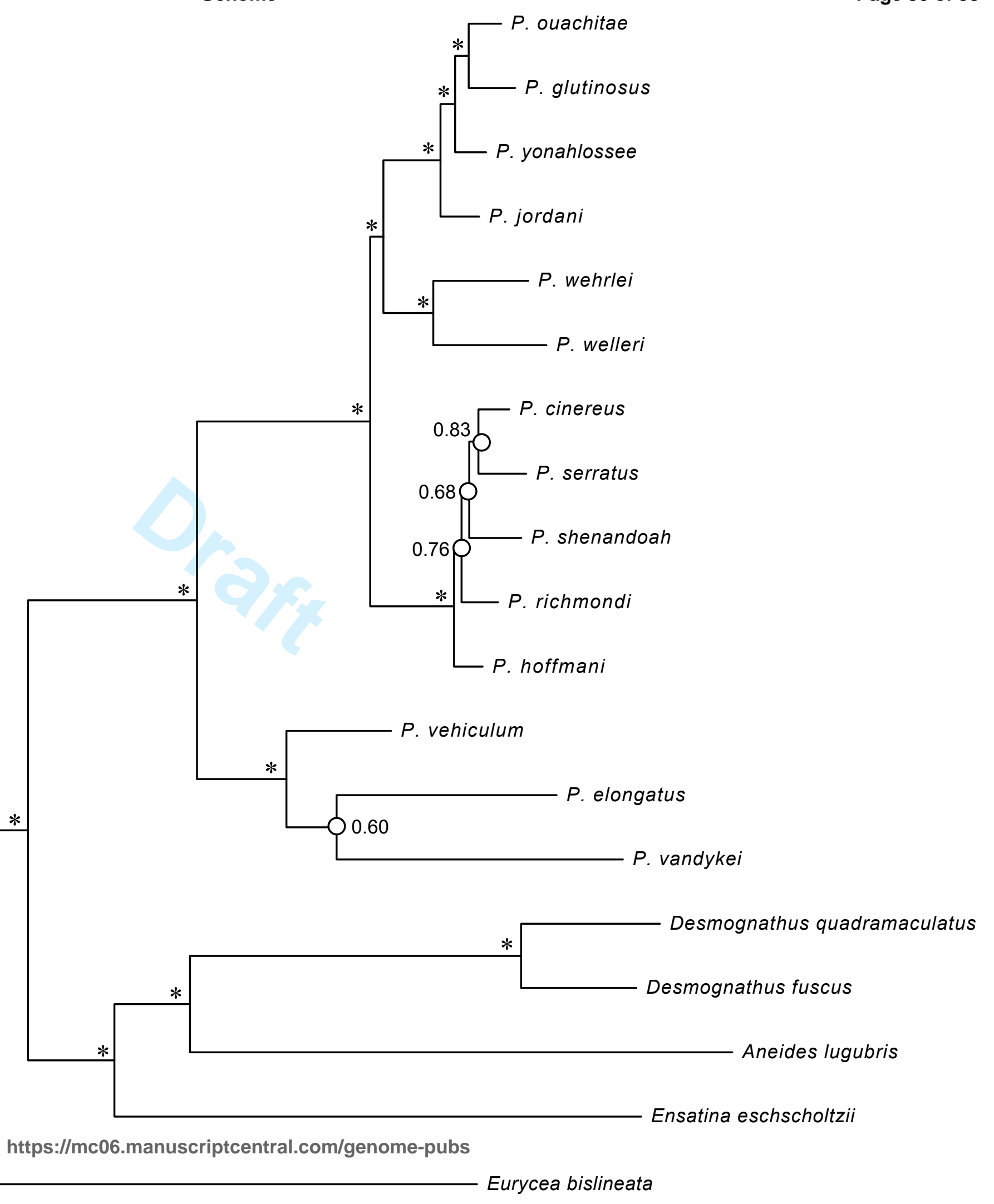




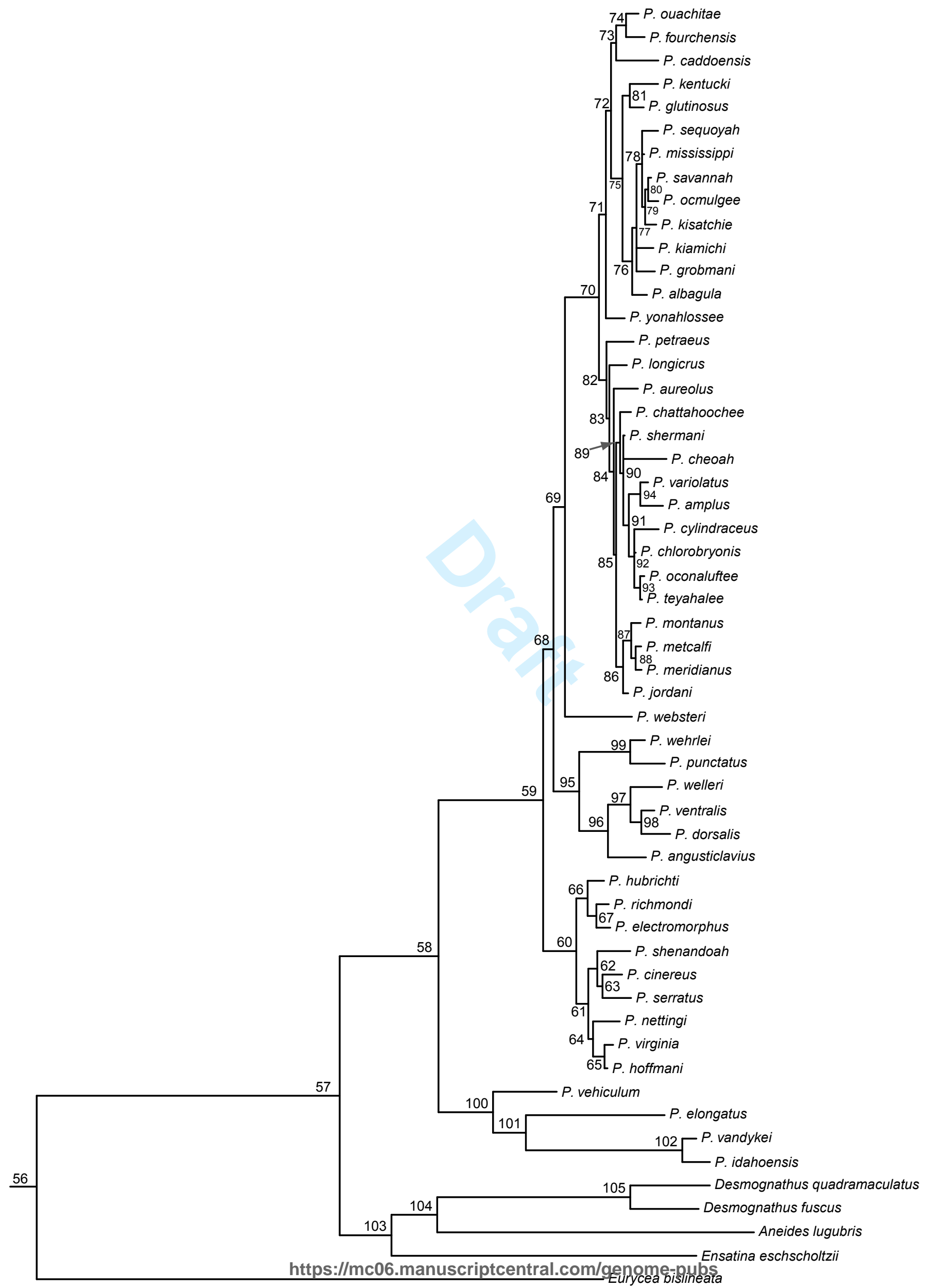




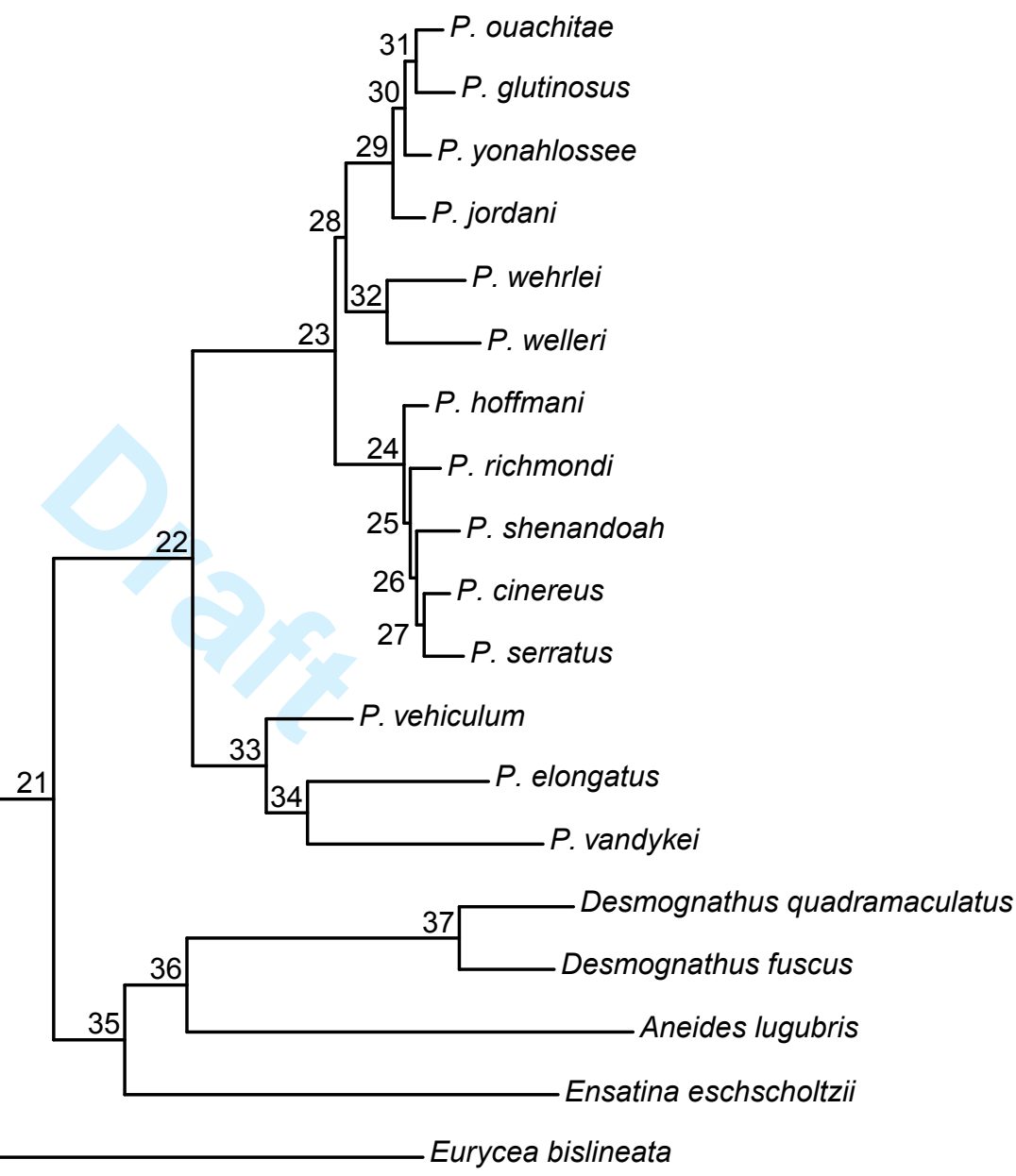


Table S1. C-values of species included in analyses.

\begin{tabular}{|c|c|c|}
\hline Species & $\begin{array}{c}\text { C-value (pg) } \\
\text { (All) }\end{array}$ & $\begin{array}{c}\text { C-value (pg) } \\
\text { (Species median) }\end{array}$ \\
\hline \multirow[t]{3}{*}{ Eurycea bislineata } & 20.75 & 24.50 \\
\hline & 24.50 & \\
\hline & 35.50 & \\
\hline \multirow[t]{4}{*}{ Aneides lugubris } & 35.68 & 42.90 \\
\hline & 42.80 & \\
\hline & 43.00 & \\
\hline & 49.60 & \\
\hline \multirow[t]{6}{*}{ Ensatina eschscholtzii } & 31.00 & 41.39 \\
\hline & 35.30 & \\
\hline & 41.27 & \\
\hline & 41.50 & \\
\hline & 42.00 & \\
\hline & 42.17 & \\
\hline \multirow[t]{3}{*}{ Desmognathus quadramaculatus } & 14.50 & 15.06 \\
\hline & 15.06 & \\
\hline & 22.00 & \\
\hline \multirow[t]{5}{*}{ Desmognathus fuscus } & 15.00 & 15.13 \\
\hline & 15.00 & \\
\hline & 15.13 & \\
\hline & 17.70 & \\
\hline & 18.00 & \\
\hline \multirow[t]{9}{*}{ Plethodon cinereus } & 20.00 & 22.64 \\
\hline & 21.40 & \\
\hline & 22.30 & \\
\hline & 22.50 & \\
\hline & 22.64 & \\
\hline & 23.00 & \\
\hline & 23.08 & \\
\hline & 26.13 & \\
\hline & 26.20 & \\
\hline \multirow[t]{4}{*}{ Plethodon elongatus } & 30.60 & 33.63 \\
\hline & 33.63 & \\
\hline & 33.63 & \\
\hline & 33.80 & \\
\hline \multirow[t]{6}{*}{ Plethodon glutinosus } & 22.50 & 28.00 \\
\hline & 25.50 & \\
\hline & 27.09 & \\
\hline & 28.00 & \\
\hline & 28.54 & \\
\hline & 28.54 & \\
\hline
\end{tabular}


43.00

$\begin{array}{lll}\text { Plethodon hoffmani } & 21.40 & 21.40 \\ \text { Plethodon jordani } & 23.10 & 27.80 \\ & 27.80 & \\ \text { Plethodon ouachitae } & 36.00 & \\ \text { Plethodon richmondi } & 33.70 & 33.70 \\ & 20.40 & 20.65 \\ \text { Plethodon serratus } & 20.90 & \\ & 22.14 & 20.43 \\ & 24.21 & \\ \text { Plethodon shenandoah } & 19.64 & \\ \text { Plethodon vandykei } & 21.07 & \\ & 19.80 & 18.20 \\ & 19.23 & 69.30 \\ \text { Plethodon vehiculum } & 18.20 & 38.05 \\ & 69.30 & \\ & 35.60 & \\ & 36.80 & 24.20 \\ & 39.30 & \\ & 40.04 & \\ & 20.30 & \\ & 28.10 & \\ & 22.60 & \\ & 25.40 & \\ & 36.10 & \\ & & \\ & & \\ & & \end{array}$


Table S2. Genetic data used in the phylogenetic analysis. Data sources by accession number prefix: JN (Fisher-Reid \& Wiens, 2011), EU (Vieites et al., 2007), FJ (Bonett et al., 2009), KR (Martin et al., 2015), DQ and AY (Wiens et al., 2006).

\begin{tabular}{|c|c|c|c|c|c|c|c|}
\hline Species & $\mathrm{BDNF}$ & GAPD & ILF3 & Mlc2a & POMC & RAG-1 & RHO \\
\hline P. albagula & - & JN798216 & JN798264 & JN798307 & - & DQ995008 & JN798363 \\
\hline P. amplus & - & JN798217 & JN798265 & JN798308 & - & DQ995010 & JN798364 \\
\hline P. angusticlavius & - & JN798218 & - & - & - & DQ995011 & - \\
\hline P. aureolus & - & JN798219 & JN798266 & JN798309 & - & DQ995012 & JN798365 \\
\hline P. caddoensis & - & JN798221 & JN798268 & JN798311 & - & DQ995013 & JN798367 \\
\hline P. chattahoochee & - & JN798222 & JN798269 & JN798312 & - & DQ995014 & JN798368 \\
\hline P. cheoah & - & JN798223 & JN798270 & JN798313 & - & DQ995015 & JN798369 \\
\hline P. chlorobryonis & - & JN798224 & JN798271 & JN798314 & - & DQ995016 & JN798370 \\
\hline P. cinereus & - & JN798228 & - & JN798317 & FJ951365 & DQ995021 & JN798374 \\
\hline P. cylindraceus & - & JN798229 & JN798272 & JN798318 & - & DQ995022 & JN798375 \\
\hline P. dorsalis & - & - & JN798273 & JN798319 & - & DQ995023 & JN798376 \\
\hline P. electromorphus & - & JN798230 & -8 & JN798320 & - & DQ995025 & JN798377 \\
\hline P. elongatus & EU275882 & JN798231 & $-O$ & $P_{-}$ & EU275836 & AY650120 & - \\
\hline P. fourchensis & EU275884 & JN798232 & JN798274 & JN798321 & EU275838 & DQ995026 & - \\
\hline P. glutinosus & - & JN798234 & - & JN798324 & - & DQ995027 & JN798379 \\
\hline P. grobmani & - & JN798236 & JN798276 & JN798325 & - & DQ995028 & JN798381 \\
\hline P. hoffmani & EU275883 & JN798238 & - & JN798327 & EU275837 & DQ995029 & JN798383 \\
\hline P. hubrichti & - & JN798239 & - & JN798328 & - & DQ995030 & JN798384 \\
\hline P. idahoensis & - & - & - & JN798329 & - & DQ995031 & - \\
\hline P. jordani & EU275881 & - & JN798278 & JN798330 & EU275835 & DQ995032 & JN798385 \\
\hline P. kentucki & - & JN798240 & JN798279 & JN798331 & - & DQ995033 & JN798386 \\
\hline P. kiamichi & - & JN798241 & JN798280 & JN798332 & - & DQ995034 & JN798387 \\
\hline P. kisatchie & - & JN798242 & JN798281 & JN798333 & - & DQ995035 & JN798388 \\
\hline P. longicrus & - & JN798243 & JN798282 & JN798334 & - & DQ995037 & JN798389 \\
\hline P. meridianus & - & JN798244 & JN798283 & JN798335 & - & DQ995038 & JN798390 \\
\hline P. metcalfi & - & JN798245 & JN798284 & - & - & DQ995039 & - \\
\hline P. mississippi & - & JN798246 & JN798285 & - & - & - & JN798391 \\
\hline P. montanus & - & JN798247 & JN798286 & JN798336 & - & DQ995043 & JN798392 \\
\hline
\end{tabular}




\begin{tabular}{|c|c|c|c|c|c|c|c|}
\hline P. netting $i$ & - & JN798248 & - & - & - & DQ995045 & JN798393 \\
\hline P. ocmulgee & - & JN798250 & JN798288 & JN798338 & - & DQ995048 & JN798395 \\
\hline P. oconaluftee & - & JN798249 & JN798287 & JN798337 & - & DQ995046 & JN798394 \\
\hline P. ouachitae & EU275877 & JN798251 & JN798289 & - & EU275831 & AY691704 & JN798396 \\
\hline P. petraeus & - & JN798252 & JN798290 & JN798339 & - & DQ995049 & JN798397 \\
\hline P. punctatus & - & JN798253 & JN798291 & - & - & DQ995050 & JN798398 \\
\hline P. richmondi & - & JN798254 & - & JN798340 & - & DQ995053 & JN798399 \\
\hline P. savannah & - & JN798255 & JN798292 & JN798341 & - & DQ995055 & JN798400 \\
\hline P. sequoyah & - & - & JN798293 & JN798342 & - & DQ995056 & JN798401 \\
\hline P. serratus & EU275876 & JN798256 & - & JN798343 & EU275830 & DQ995057 & JN798402 \\
\hline P. shenandoah & - & JN798257 & - & JN798344 & - & DQ995062 & JN798403 \\
\hline P. shermani & - & JN798259 & JN798294 & JN798346 & - & DQ995065 & JN798405 \\
\hline P. teyahalee & EU275880 & - & JN798295 & JN798347 & EU275834 & DQ995068 & JN798406 \\
\hline P. vandykei & EU275879 & JN798260 & - & - & EU275833 & AY691715 & - \\
\hline P. variolatus & - & - & JN798296 & JN798348 & - & DQ995070 & JN798407 \\
\hline P. vehiculum & - & - & $-O$ & JN798349 & - & AY691716 & JN798408 \\
\hline P. ventralis & - & JN798261 & JN798297 & JN798350 & - & DQ995071 & JN798409 \\
\hline$P$. virginia & - & - & JN798298 & JN798351 & - & DQ995072 & JN798410 \\
\hline P. websteri & - & - & JN798299 & JN798352 & - & DQ995073 & JN798411 \\
\hline$P$. wehrlei & - & - & JN798300 & JN798353 & - & DQ995075 & JN798412 \\
\hline P. welleri & - & JN798262 & JN798301 & - & - & AY691717 & JN798413 \\
\hline P. yonahlossee & EU275878 & JN798263 & JN798302 & JN798354 & EU275832 & DQ995077 & JN798414 \\
\hline Eurycea bislineata & EU275861 & - & - & - & EU275815 & AY691706 & JN798360 \\
\hline Aneides lugubris & EU275893 & - & - & - & EU275847 & AY650118 & JN798356 \\
\hline Ensatina eschscholtzii & EU275862 & - & - & - & EU275816 & EU275785 & JN798361 \\
\hline Desmognathus quadramaculatus & - & - & - & - & KR732359 & AY650117 & - \\
\hline Desmognathus fuscus & EU275858 & - & - & - & EU275812 & EU275781 & - \\
\hline
\end{tabular}


Table S3. C-values estimated by ACR for all nodes on the full and known-only phylogenies. Node numbers correspond to Figs. S1, S2.

\begin{tabular}{|c|c|c|c|}
\hline $\begin{array}{c}\text { Node } \\
\text { (all, known) }\end{array}$ & Clade Name & $\begin{array}{c}\text { C-value (pg) } \\
\text { (All) }\end{array}$ & $\begin{array}{c}\text { C-value (pg) } \\
\text { (Only known) }\end{array}$ \\
\hline 56,20 & (Root) & 29.80 & 29.88 \\
\hline 57,21 & Plethodontinae & 32.63 & 32.77 \\
\hline 58,22 & Plethodon & 33.82 & 34.09 \\
\hline 59,23 & Eastern Plethodon & 25.71 & 26.37 \\
\hline 60,24 & P. cinereus group & 22.22 & 21.65 \\
\hline 61 & & 21.54 & \\
\hline 62,26 & & 21.10 & 21.06 \\
\hline 63,27 & & 21.29 & 21.30 \\
\hline 64 & & 21.51 & \\
\hline 65 & & 21.42 & \\
\hline 66 & & 21.67 & \\
\hline 67 & & 21.25 & \\
\hline 68,28 & & 25.99 & 26.52 \\
\hline 69 & $P$. glutinosus group $+P$. websteri & 26.77 & \\
\hline 70,29 & P. glutinosus group & 29.07 & 28.90 \\
\hline 71,30 & & 29.84 & 29.93 \\
\hline 72,31 & & 30.16 & 30.52 \\
\hline 73 & & 30.82 & \\
\hline 74 & & 32.09 & \\
\hline 75 & & 29.40 & \\
\hline 76 & & 29.40 & \\
\hline 77 & & 29.40 & \\
\hline 78 & & 29.40 & \\
\hline 79 & & 29.40 & \\
\hline 80 & & 29.40 & \\
\hline 81 & & 28.92 & \\
\hline 82 & & 28.74 & \\
\hline 83 & & 28.62 & \\
\hline 84 & & 28.43 & \\
\hline 85 & & 28.32 & \\
\hline 86 & & 28.02 & \\
\hline 87 & & 28.02 & \\
\hline 88 & & 28.02 & \\
\hline 89 & & 28.32 & \\
\hline 90 & & 28.32 & \\
\hline 91 & & 28.32 & \\
\hline 92 & & 28.32 & \\
\hline
\end{tabular}




$\begin{array}{cl}93 & \\ 94 & \\ 95,32 & \text { P. welleri \& P. wehr } \\ 96 & \\ 97 & \\ 98 & \\ 99 & \\ 100,33 & \text { Western Plethodon } \\ 101,34 & \\ 102 & \\ 103,35 & \\ 104,36 & \\ 105,37 & \\ -, 25 & \end{array}$

28.32

28.32

24.96

25.02

24.14

23.50

23.50

24.37

38.71

38.77

41.99

41.75

67.04

32.49

32.57

31.03

31.14

17.65

17.66

21.29 